\title{
"Craving": Exploring the Components of the Desires for Alcohol Questionnaire (DAQ) and the Relation to the Severity of Alcohol Problems
}

\author{
SONJA C. PASCHE, M.PSYCH., ${ }^{a}$ MATTHEW GARNER, PH.D., ${ }^{b, c}$ DAVID S. BALDWIN, D.M., FR.PSYCH., ${ }^{a, c}$ AND \\ JULIA M. A. SINCLAIR, M.R.C.PSYCH., D.PHIL. ${ }^{c, *}$ \\ ${ }^{a}$ Department of Psychiatry and Mental Health, University of Cape Town, Cape Town, South Africa \\ ${ }^{b}$ Faculty of Social and Human Sciences, University of Southampton, Southampton, United Kingdom \\ ${ }^{c}$ Faculty of Medicine, University of Southampton, Southampton, United Kingdom
}

\begin{abstract}
Objective: The aims of this study were (a) to explore the components of craving, as measured by the Desires for Alcohol Questionnaire (DAQ), and (b) to examine how craving may relate to the severity of alcohol problems. Method: A total of 106 patients seeking treatment for an alcohol use disorder (AUD) completed the DAQ and Alcohol Use Disorders Identification Test (AUDIT). The overall sample was predominantly male $(63 \%)$ with a mean age of 44 years. Sixty-one percent of the participants were abstinent from alcohol at the time of the study. Principal components analysis was conducted on the DAQ for the overall, abstinent, and currently drinking samples. Correlations were computed between the DAQ and AUDIT scores, and differences in craving between the abstinent and currently drinking samples were
\end{abstract}

investigated. Results: Components of craving, as measured by the DAQ, included the desire to drink, the ability to control drinking, positive reinforcement and negative reinforcement. Drinkers displayed stronger cravings $(M d n=47.00$, interquartile range $[\mathrm{IQR}]=32.0-65.0)$ than those currently abstinent $(M d n=33.00, \mathrm{IQR}=26.0-43.0 ; U=850.0$; $z=3.127, p<.01 ; r=.30)$. The intensity of craving increased with the severity of the AUD in current drinkers $(r=.739, p<.001)$. Conclusions: Because of the small sample size, the results of the study should be regarded as preliminary. The components of craving, as measured by the DAQ, support those previously identified in the literature. The study supports the notion that craving is positively associated with the severity of an AUD. (J. Stud. Alcohol Drugs, 74, 000-000, 2013)
$\mathrm{C}$ RAVING IS REGARDED as a central component in understanding alcohol or other drug dependence. It is listed as a key symptom for alcohol dependence syndrome in the International Classification of Diseases, where it is described as a "strong desire to take" alcohol (World Health Organization, 1992). Craving has been found to increase with the severity of alcohol use disorders (AUDs) (Bohn et al., 1995). There is also a difference in the intensity of cravings for alcohol between individuals with and without an AUD. Individuals with an AUD scored significantly higher on the Desires for Alcohol Questionnaire (DAQ), a measure of craving, than those individuals without an AUD (Kramer et al., 2010). Craving is also considered to predict relapse (e.g., Flannery et al., 2001), although this notion has been criticized (Drummond, 2001; Drummond et al., 2000).

Despite the emphasis on craving in the substance dependence literature, there is a lack of consensus regarding its

Received: January 30, 2013. Revision: April 10, 2013.

This study was supported by a grant from the Research Management Committee of the University of Southampton. SCP was supported through the European Union Marie Curie (People) International Research Staff Exchange Scheme (PIRSES-GA-2010-269213: 'EUSARNAD').

*Correspondence may be sent to Julia M. A. Sinclair, Senior Lecturer in Psychiatry, University Department of Psychiatry, Academic Centre, College Keep, 4-12 Terminus Terrace, Southampton SO14 3DT, United Kingdom, or via email at: julia.sinclair@soton.ac.uk. definition (Flannery et al., 2001). Craving is conceptualized in a number of ways by cognitive, phenomenological, and conditioning models that attempt to explain its nature (Drummond, 2001). For example, craving has been differentiated as to whether it arises in response to withdrawal symptoms, cues, lack of pleasure, or hedonic desires (Wright et al., 1993). This has led to the suggestion that different groups of patients have different mechanisms that promote alcohol craving (Addolorato et al., 2005).

The DAQ attempts to measure craving for alcohol by incorporating both a measure of "the urge to drink" as well as the anticipated outcomes from drinking (Love et al., 1998). Previous research indicates that the DAQ consists of three or four dimensions, namely "strong desires/intentions to drink," "negative reinforcement," "positive reinforcement," and "the ability to control drinking" (Kramer et al., 2010; Love et al., 1998). These dimensions reflect the complexity of the concept of "craving" beyond the "urge to drink." This study examines the components of the DAQ, as well as its relation to the severity of AUDs. It is hoped that this will extend our understanding of the concept of craving as well as provide a comparison with components identified in previous investigations (e.g., Kramer et al., 2010; Love et al., 1998).

This study had the following objectives: (a) to explore the components of craving, as measured by the DAQ, and (b) to explore how components of craving (as measured by the DAQ) relate to the severity of alcohol problems. 


\section{Method}

\section{Subjects}

Patients seeking assistance for their AUD were recruited from community alcohol services located in Hampshire, UK. This was a cross-sectional survey of all patients in treatment, and key workers were asked to inform all of their patients about the study and provide them with the patient information sheet and the option to participate. Because they have a chronic relapsing condition, patients with AUDs often come into and go out of treatment as required; therefore, treatment time was not defined. Patients who wished to participate gave informed consent, were assured that they were able to withdraw from the study at any stage, and were assured that choosing not to participate in the study would not negatively affect future treatment. All patients able to give informed consent were eligible for the study, whether they were still currently drinking or whether they were in the relapse-prevention phase of treatment ("abstinent"). Other than medical detoxification, the treatment modality was a mixed psychosocial program (including group and individual work). Anticraving medication and disulfiram were not funded for use and, therefore, were rarely prescribed. Abstinence was self-reported and confirmed via breath alcohol tests at treatment sessions. Of 123 participants recruited for the study, 106 completed the DAQ and the Alcohol Use Disorders Identification Test (AUDIT). Ethics approval was granted by the Southampton Research Ethics Committee (REC ref:08/H0502/40).

\section{Instruments}

Participants completed a series of questionnaires and tasks, including the AUDIT as a measure of the severity of alcohol use problems, the DAQ as a measure of craving, and a structured form requesting demographic details.

The DAQ, used as a measure of craving, was originally conceptualized as a 36-item questionnaire but has since been reduced to a 14-item version (Kramer et al., 2010; Love et al., 1998). The 14-item version was used in this study, with responses reflected on a 7-point Likert scale. The 14-item DAQ has demonstrated good reliability, with Cronbach's $\alpha$ ranging from .88 to .93 (Kramer et al., 2010). Items 11 and 12 were reversed, so that an increase in the DAQ score (and all individual items) corresponds with an increase in craving.

The AUDIT is a 10-item questionnaire that assists with screening for AUDs. Increasing scores indicate more problematic alcohol use, with scores greater than 20 indicating alcohol dependence (Babor et al., 2001). The AUDIT has been internationally validated, providing an accurate measure of risk across various cultures (Babor et al., 2001). It is used to assess the severity of alcohol misuse in the sample.
Analyses

Principal component analyses (PCAs). PCAs were conducted on the overall sample, as well as separately in the abstinent and currently drinking groups. Only those participants who answered all items of the DAQ were included, reducing the overall sample to 106 . For each analysis, PCA was conducted on the 14 items of the DAQ using oblique rotation (direct oblimin), as it was expected that the factors composing the DAQ would correlate (Kramer et al., 2010). The Kaiser-Meyer-Olkin (KMO) measure verified the sampling adequacy for all analyses, with scores for all three analyses well above the recommended cutoff of 0.5 (Field, 2009). The KMO scores for overall, abstinent, and currently drinking samples were $.849, .732$ and .756 , respectively. Bartlett's test of sphericity indicated that correlations between items were sufficiently large for PCA $(p<.001$ for all three samples). As taken from the reproduced correlations table, the percentage of nonredundant residuals was less than $50 \%$ for all three samples $(48 \%, 34 \%$, and $32 \%$ for overall, abstinent, and currently drinking samples, respectively). Components were identified using Kaiser's criterion of eigenvalues greater than 1 and by the inspection of scree plots. To be considered part of a component, items were required to have a factor loading of .4 or greater. If any item had factor loadings of .4 for more than one component, it was considered part of the component for which it had the highest factor loading. Cronbach's $\alpha$ was calculated to measure the reliability of the DAQ. For this purpose, Items 11 and 12 of the DAQ were reverse scored, as they are negatively phrased.

Correlations between the DAQ and AUDIT scores were calculated for the overall, abstinent, and currently drinking samples. The difference in DAQ scores between the abstinent and currently drinking samples was investigated using the Mann-Whitney test.

\section{Results}

The overall sample $(N=106)$ consisted of a greater proportion of men $(n=76,63 \%)$ than women $(n=39,37 \%)$, with a mean age of 44.2 years $(S D=11.6)$. The majority of participants were not working at the time of the study $(n=$ $84,79 \%$ ) and had at least one family member with a history of an AUD ( $n=68$ of $N=105,65 \%)$. Participants had suffered with an AUD for a median of 15.4 years (interquartile range $[\mathrm{IQR}]=6.0-23.3)$ and had undergone a median of one detoxification $(\mathrm{IQR}=0.0-2.0)$.

The majority of the sample was abstinent at the time of the study $(n=65,61 \%)$, with abstinent participants having a median of 49 days of abstinence (IQR $=28.0-120.0 ; M$ $=90.2, S D=120.7)$. The abstinent sample had a median AUDIT score of 33 (IQR $=29.0-37.0 ; M=30.5, S D=$ 9.4) compared with a median AUDIT score of 29 (IQR = 26.0-34.5; $M=29.4, S D=7.4$ ) for the currently drinking 
TABLE 1. Desires for Alcohol Questionnaire (DAQ) component solutions

\begin{tabular}{|c|c|c|c|c|c|c|c|c|c|c|c|}
\hline \multirow[b]{2}{*}{ DAQ item number } & \multicolumn{3}{|c|}{ Total sample $(N=106)$} & \multicolumn{4}{|c|}{ Abstinent sample $(n=65)$} & \multicolumn{4}{|c|}{ Currently drinking sample $(n=41)$} \\
\hline & 1 & 2 & 3 & 1 & 2 & 3 & 4 & 1 & 2 & 3 & 4 \\
\hline \multicolumn{12}{|l|}{ 1. I want a drink so much } \\
\hline I can almost taste it. & .901 & -.175 & -.017 & .689 & -.275 & .119 & -.219 & .995 & -.052 & -.037 & -.128 \\
\hline $\begin{array}{l}\text { 2. My desire to drink now } \\
\text { seems overwhelming. }\end{array}$ & 848 & -126 & 036 & & -129 & & & & & & \\
\hline 3. I would do almost anything & .848 & -.120 & .036 & .804 & -.129 & .018 & -.035 & .892 & .033 & -.008 & .101 \\
\hline to have a drink now. & .750 & .003 & .117 & .883 & .076 & .017 & .003 & .637 & .187 & .005 & .349 \\
\hline $\begin{array}{l}\text { 4. I am going to drink as } \\
\text { soon as I possibly can. } \\
\text { 5. I would consider having }\end{array}$ & .891 & -.042 & -.003 & .941 & .140 & .017 & .129 & .725 & -.119 & .161 & .116 \\
\hline a drink now. & .829 & -.011 & .104 & .790 & .038 & .177 & .038 & .660 & .015 & .196 & .209 \\
\hline $\begin{array}{l}\text { 6. I would accept a drink now if } \\
\text { it was offered to me. }\end{array}$ & .908 & .002 & -.041 & .740 & -.016 & -.055 & -.184 & .807 & -.033 & .203 & -.022 \\
\hline $\begin{array}{l}\text { 7. I would feel as if all the bad } \\
\text { things in my life had } \\
\text { disappeared if I }\end{array}$ & & & & & & & & & & & \\
\hline $\begin{array}{l}\text { drank now. } \\
\text { 8. Even major problems in my }\end{array}$ & .023 & -.086 & .831 & .090 & .014 & .857 & .110 & .316 & -.091 & -.136 & .650 \\
\hline $\begin{array}{l}\text { life would not bother me } \\
\text { if I drank now. }\end{array}$ & -.046 & -.021 & .894 & .150 & .051 & .791 & -.030 & .062 & -.006 & -.141 & .875 \\
\hline 9. I would feel less worried & & & & & & & & & & & \\
\hline $\begin{array}{l}\text { if I drank now. } \\
\text { 10. Drinking now would }\end{array}$ & .002 & .052 & .882 & .014 & .057 & .940 & -.007 & .087 & -.070 & .171 & .623 \\
\hline $\begin{array}{l}\text { make me feel less tense. } \\
\text { 11. If I started drinking now }\end{array}$ & .122 & .031 & .735 & -.047 & -.161 & .599 & -.497 & -.098 & -.031 & .304 & .768 \\
\hline $\begin{array}{l}\text { I would be able to stop. } \\
\text { 12. I could easily limit how } \\
\text { much I would drink if }\end{array}$ & -.097 & .899 & .117 & -.130 & .887 & .181 & -.057 & -.082 & .939 & .160 & .117 \\
\hline I drank now. & .024 & .857 & -.160 & .138 & .874 & -.119 & -.095 & .074 & .846 & -.157 & -.217 \\
\hline $\begin{array}{l}\text { 13. Drinking would be } \\
\text { satisfying now. }\end{array}$ & .551 & .358 & .197 & .103 & .126 & .129 & -.830 & .068 & .045 & .916 & .051 \\
\hline $\begin{array}{l}\text { 14. Drinking would be } \\
\text { pleasant now. }\end{array}$ & .686 & .301 & -.019 & .064 & .104 & -.110 & -.911 & .217 & -.008 & .849 & -.044 \\
\hline Eigenvalues & 6.777 & 1.897 & 5.383 & 5.739 & 1.790 & 4.538 & 3.366 & 6.359 & 1.903 & 3.834 & 5.043 \\
\hline Cronbach's $\alpha$ & .930 & .749 & .869 & .912 & .702 & .887 & .885 & .942 & .788 & .934 & .826 \\
\hline
\end{tabular}

Note: Bold indicates factors that cluster together.

sample. Those participants currently drinking drank a median of 7 days a week (IQR $=4.0-7.0 ; M=5.6, S D=2.3$ ) and consumed a median of 18 units of alcohol a day (IQR = 9.5-30.0; $M=23.2, S D=25.2$ ).

\section{Principal component analyses}

Total sample. Following oblimin rotation, three components were identified as central to the DAQ. Items clustering together suggest the following three components: (a) desire to drink and positive reinforcement (Items 1-6, 13, 14); (b) ability to control drinking (Items 11, 12); (c) negative reinforcement (Items 7-10). Cronbach's $\alpha$ for these three components was $.930, .749$, and .869 , and .902 for the overall DAQ, indicating good reliability for the overall scale and subscales. Intercorrelations among the three components were .110 (Components 1 and 2), .035 (Components 2 and 3 ), and .633 (Components 1 and 3).

Abstinent sample. Four components were identified in the abstinent subsample. Compared with the total sample, Items 13 and 14 no longer formed part of the first component, making up a separate dimension. The components were as follows: (a) desire to drink (Items 1-6); (b) ability to control drinking (Items 11, 12); (c) negative reinforcement (Items 7-10); (d) positive reinforcement (Items 13, 14). Cronbach's $\alpha$ for the overall (abstinent) sample was .864 and for Components 1-4 was .912,.702, .887, and .885, respectively. Intercorrelations among the components ranged from .009 (Components 2 and 3) to .498 (Components 1 and 3).

Currently drinking sample. The four components identified in the currently drinking sample were identical to those for the abstinent sample. Cronbach's $\alpha$ for the total sample was .922 and for Components $1-4$ was $.942, .788, .934$, and .826 , respectively. Intercorrelations among the components ranged from -.001 (Components 2 and 3) to .544 (Components 1 and 4) (Table 1).

\section{Correlations and differences between abstinent and currently drinking samples}

There was a significant relationship between the total DAQ and total AUDIT score for the overall $\left(r_{s}=.300, p<\right.$ 
TABLE 2. Correlations between Desires for Alcohol (DAQ) component and AUDIT scores

\begin{tabular}{lcc}
\hline & \multicolumn{2}{c}{ AUDIT } \\
\cline { 2 - 3 } & $\begin{array}{c}\text { Abstinent } \\
\text { sample } \\
r_{s}\end{array}$ & $\begin{array}{c}\text { Currently } \\
\text { drinking } \\
\text { sample } \\
r_{s}\end{array}$ \\
$\begin{array}{l}\text { Variable } \\
\begin{array}{l}\text { DAQ Component 1 } \\
\text { "Desire to drink" }\end{array}\end{array}$ & .099 \\
$\begin{array}{l}\text { DAQ Component 2 } \\
\text { "Ability to control drinking" }\end{array}$ & $.346 * *$ \\
$\begin{array}{l}\text { DAQ Component 3 } \\
\text { "Negative reinforcement" }\end{array}$ & -.054 & .270 \\
$\begin{array}{l}\text { DAQ Component 4 } \\
\text { "Positive reinforcement" }\end{array}$ & .080 & $.597 * * *$ \\
\hline
\end{tabular}

Note: AUDIT $=$ Alcohol Use Disorders Identification Test.

${ }^{*} p<.05 ;{ }^{* *} p<.01 ; * * p<.001$.

$.01)$ and currently drinking $\left(r_{s}=.739, p<.001\right)$ samples. The correlation between the DAQ and AUDIT scores for the abstinent sample was nonsignificant $\left(r_{s}=.142, p=\right.$ N.s. $)$.

For the overall sample, the total AUDIT score was significantly correlated with Component 1 (desire to drink and positive reinforcement) $\left(r_{s}=.277, p<.01\right)$ and Component 2 (ability to control drinking) $\left(r_{s}=.318, p<.01\right)$ of the DAQ. However, there was no significant relationship between the AUDIT score and Component 3 (negative reinforcement) $\left(r_{s}\right.$ $=.157, p=$ N.s.). The correlations between the total AUDIT score and the DAQ component scores for the abstinent and currently drinking groups are displayed in Table 2. AUDIT scores are significantly positively associated with Components 1,3 , and 4 (desire to drink, negative reinforcement, and positive reinforcement) for the currently drinking group. In comparison, there is no significant relationship between these three components and the AUDIT score for the abstinent group. However, the abstinent sample displays a significant correlation between the AUDIT score and Component 2 (the ability to control drinking), which is not the case for

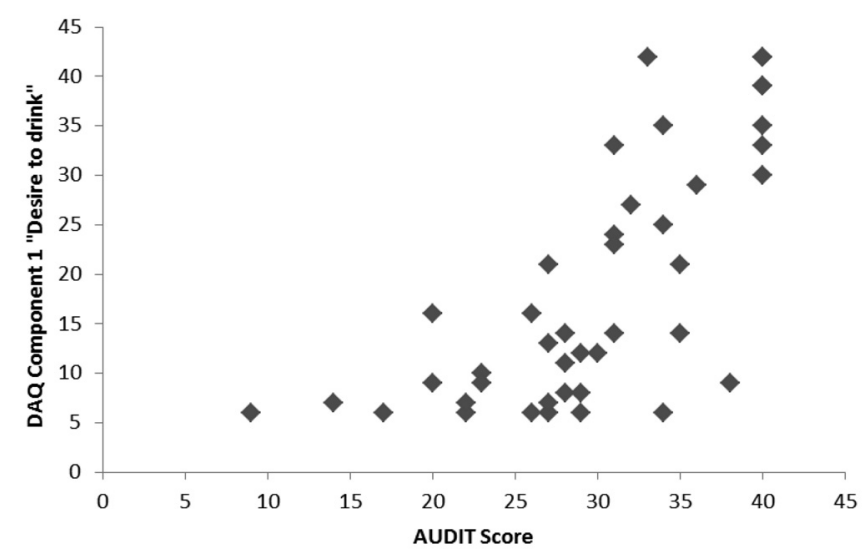

FIGURE 1. Scatter plot for currently drinking sample: Desires for Alcohol (DAQ) Component 1 and Alcohol Use Disorders Identification Test (AUDIT) score the currently drinking sample. This correlation suggests that as the severity of the AUD increases, the abstinent sample reports less ability to control drinking (if they were to do so) (Table 2; Figures 1-4).

The difference in DAQ scores between the abstinent and currently drinking samples was statistically significant $(U$ $=850.0 ; z=3.127, p=.002 ; r=.30)$, with drinkers having greater cravings $(M d n=47.00, \mathrm{IQR}=32.0-65.0)$ than those currently abstinent $(M d n=33.00, \mathrm{IQR}=26.0-43.0)$. The scores obtained on the four components of the DAQ also differed significantly between the abstinent and currently drinking groups (Table 3 ). The currently drinking group displayed a greater desire to drink and experienced greater positive and negative reinforcement for their drinking behavior. Contrary to expectations, the currently drinking group endorsed slightly better control over their drinking than the abstinent group (although this may represent continued denial of the nature of their problem). However, once the Bonferroni correction was made, no difference remained for Component 2 (ability to control drinking) at a significance level of $p<.01$. [COMP: Table 3 about here]

\section{Discussion}

When analyzed separately, four identical components were found in both the abstinent and currently drinking samples, namely "desire to drink" (Items 1-6), "ability to control drinking" (Items 11, 12), "negative reinforcement" (Items 7-10), and "positive reinforcement" (Items 13, 14). This supports the findings of Love et al. (1998), who found the same factors, composed of the same items, in a sample of 126 patients with AUDs. It is not clear why, when analyzed as a whole, the overall sample produces three components, instead of the four when the subsamples were analyzed separately. This may reflect the differences in factor structure (and perhaps sample size) observed between the

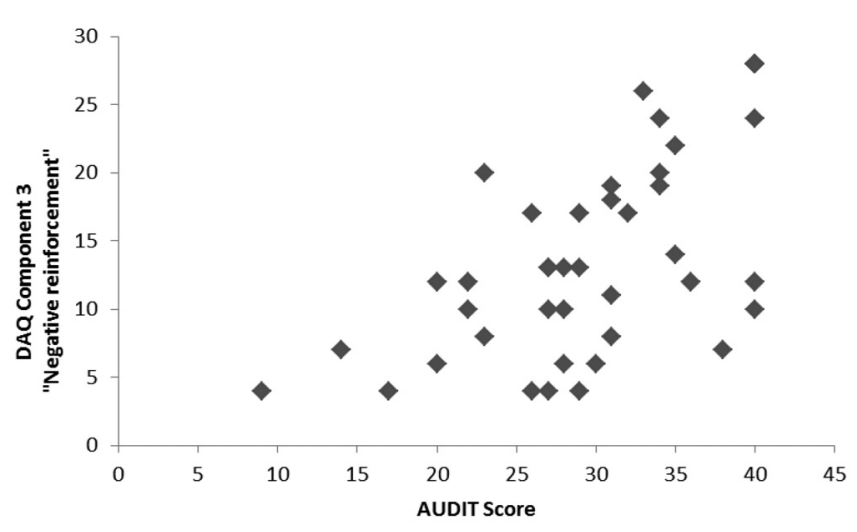

FIGURE 2. Scatter plot for currently drinking sample: Desires for Alcohol (DAQ) Component 3 and Alcohol Use Disorders Identification Test (AUDIT) score 


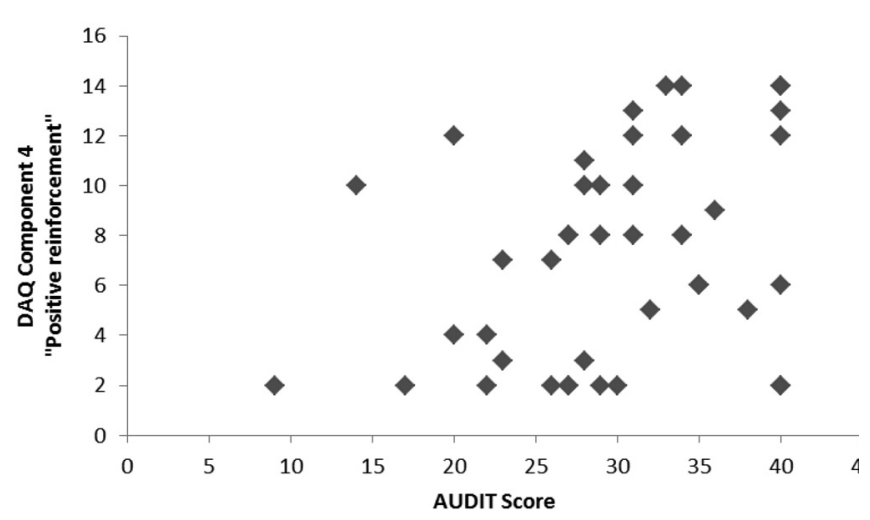

FiguRE 3. Scatter plot for currently drinking sample: Desires for Alcohol (DAQ) Component 4 and Alcohol Use Disorders Identification Test (AUDIT) score

abstinent and currently drinking subgroups. For the overall sample, the "desire to drink" and "positive reinforcement" factors joined to become one factor, with the composition of the other two factors remaining the same. Kramer et al. (2010) also uncovered a three-component structure for both AUD and non-AUD samples, although in their research the "positive reinforcement "and "ability to control drinking" components merged, in contrast to the findings of this study.

It is possible that these variations may be attributed to the differences in sample size, with Kramer et al.'s (2010) study consisting of considerably larger sample sizes $(N=2,960)$ than those in the present and Love et al.'s (1998) studies. In the present and Love et al.'s (1998) study, the DAQ items are reflected in the present tense, whereas those in Kramer et al.'s article (2010) were rephrased in the past tense - this may account for different findings across studies.

Our findings suggest that craving is linked to the presence and severity of an AUD, as a significant positive correlation between the overall AUDIT and DAQ scores was found. This correlation was large for current drinkers with an AUD but was nonsignificant for the abstinent group. Current drinkers had greater cravings than those individuals who were

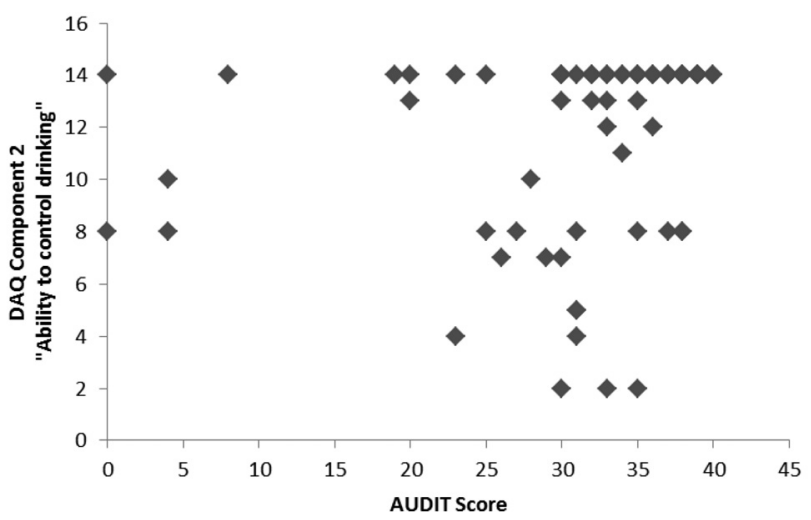

FiguRE 4. Scatter plot for abstinent sample: Desires for Alcohol (DAQ) Component 2 and Alcohol Use Disorders Identification Test (AUDIT) score

abstinent from alcohol. These results are consistent with previous research (Bohn et al., 1995; Kramer et al., 2010), which supports the notion that craving is positively linked to the current severity of alcohol use problems, but further demonstrates that once a person is abstinent from alcohol, cravings are significantly reduced.

An analysis of the components of craving, as measured by the DAQ, illustrated significant differences between the abstinent and currently drinking groups. In particular, the currently drinking group displayed a greater desire to drink and more reinforcement (both positive and negative) for their drinking behavior than the abstinent group. The increase in the severity of an AUD was also positively associated with these components of craving for the currently drinking, but not the abstinent, group. For the abstinent group, an increase in the severity of their AUD was associated with a recognition that they had a decreased ability to control drinking. Implications for treatment may be that increasing severity of an AUD requires patients to recognize their lack of control over their drinking to achieve abstinence; however, as this was a cross-sectional sample, it is not possible to determine the direction of causality for this association. Similarly, it may

TABLE 3. Differences in Desire for Alcohol (DAQ) component scores for abstinent and currently drinking groups

\begin{tabular}{|c|c|c|c|c|c|}
\hline Variable & $U$ & $z$ & $r$ & $\begin{array}{c}M d n \\
\text { (IQR) for } \\
\text { abstinent }\end{array}$ & $\begin{array}{l}M d n \\
\text { (IQR) for } \\
\text { currently } \\
\text { drinking }\end{array}$ \\
\hline $\begin{array}{l}\text { DAQ Component } 1 \text { (Items 1-6) } \\
\text { "Desire to drink" }\end{array}$ & 728.5 & $4.14 * * *$ & .40 & $\begin{array}{c}7.0 \\
(6.0-11.0)\end{array}$ & $\begin{array}{c}14.0 \\
(8.0-29.0)\end{array}$ \\
\hline $\begin{array}{l}\text { DAQ Component } 2 \text { (Items 11, 12) } \\
\text { "Ability to control drinking" }\end{array}$ & 994.5 & $2.40^{*}$ & -.23 & $\begin{array}{c}14.0 \\
(8.0-14.0)\end{array}$ & $\begin{array}{c}11.0 \\
(8.0-14.0)\end{array}$ \\
\hline $\begin{array}{l}\text { DAQ Component } 3 \text { (Items 7-10) } \\
\text { "Negative reinforcement" }\end{array}$ & 965.5 & $2.65 * *$ & .26 & $\begin{array}{c}8.0 \\
(4.0-16.0)\end{array}$ & $\begin{array}{c}12.5 \\
(8.00-19.00)\end{array}$ \\
\hline $\begin{array}{l}\text { DAQ Component } 4 \text { (Items 13, 14) } \\
\text { "Positive reinforcement" }\end{array}$ & 909.0 & $3.10 * *$ & .30 & $\begin{array}{c}3.0 \\
(2.0-7.0)\end{array}$ & $\begin{array}{c}7.5 \\
(3.0-11.00)\end{array}$ \\
\hline
\end{tabular}

Note: $\mathrm{IQR}=$ interquartile range.

$* p<.05 ; * * p<.01 ; * * * p<.001$. 
be that the salience of reinforcement for drinking behavior among individuals currently drinking could be addressed to help personalize treatment strategies. These interpretations would support accepted treatment modalities such as cognitive-behavioral therapy, as well as more recent pharmacological therapies aimed at reducing alcohol consumption in alcohol-dependent patients (Schuckit, 2009) and the need for more intensive forms of treatment for those individuals with more severe AUDs (e.g. Babor et al., 2001; Rychtarik et al., 2000).

The study has a number of limitations, of which the small sample size is a key consideration. The small sample size may account for the difference in the number of components identified between the overall sample and the subsamples (the abstinent and currently drinking group). The results should therefore be considered preliminary, and it is recommended that future research with larger samples be conducted.

\section{Conclusion}

This research highlights the multidimensional nature of craving, as measured by the DAQ. Craving relates to the desire to drink and the ability to control the impulse to drink, as well as positive (e.g., pleasant experience of drinking) and negative (e.g., as a way to forget about problems) reinforcement resulting from drinking. The DAQ had high internal reliability, corroborating previous research findings. The intensity of craving increases with the severity of alcohol problems among individuals with an AUD and distinguishes between current drinkers and those who are abstinent from alcohol.

\section{Acknowledgments}

The authors thank Tom Wood and Katharine Reed for data collection in this study and Bina Nausheen for assisting in the development of the protocol. We are grateful to the staff of the local Community Alcohol Services in Hampshire and all patients who gave of their time to participate.

\section{References}

Addolorato, G., Leggio, L., Abenavoli, L., \& Gasbarrini, G., \& the Alcoholism Treatment Study Group. (2005). Neurobiochemical and clinical aspects of craving in alcohol addiction: A review. Addictive Behaviors, 30, 1209-1224.

Babor, T. F., Higgins-Biddle, J. C., Saunders, J. B., \& Monteiro, M. G. (2001). AUDIT. The Alcohol Use Disorders Identification Test. Guidelines for use in primary care (2nd ed.). Geneva, Switzerland: World Health Organization.

Bohn, M. J., Krahn, D. D., \& Staehler, B. A. (1995). Development and initial validation of a measure of drinking urges in abstinent alcoholics. Alcoholism: Clinical and Experimental Research, 19, 600-606.

Drummond, D. C. (2001). Theories of drug craving, ancient and modern. Addiction, 96, 33-46.

Drummond, D. C., Litten, R. Z., Lowman, C., \& Hunt, W. A. (2000). Craving research: Future directions. Addiction, 95, Supplement 2, S247-S255.

Field, A. (2009). Discovering statistics using SPSS (3rd ed.). London, England: Sage.

Flannery, B. A., Roberts, A. J., Cooney, N., Swift, R. M., Anton, R. F., \& Rohsenow, D. J. (2001). The role of craving in alcohol use, dependence, and treatment. Alcoholism: Clinical and Experimental Research, 25, 299-308.

Kramer, J. R., Chan, G., Hesselbrock, V. M., Kuperman, S., Bucholz, K. K., Edenberg, H. J., . . Porjesz, B. (2010). A principal components analysis of the abbreviated Desires for Alcohol Questionnaire (DAQ). Journal of Studies on Alcohol and Drugs, 71, 150-155.

Love, A., James, D., \& Willner, P. (1998). A comparison of two alcohol craving questionnaires. Addiction, 93, 1091-1102.

Rychtarik, R. G., Connors, G. J., Whitney, R. B., McGillicuddy, N. B., Fitterling, J. M., \& Wirtz, P. W. (2000). Treatment settings for persons with alcoholism: Evidence for matching clients to inpatient versus outpatient care. Journal of Consulting and Clinical Psychology, 68, 277-289.

Schuckit, M. A. (2009). Alcohol-use disorders. The Lancet, 373, 492-501.

World Health Organization. (1992). International statistical classification of diseases and related health problems. Geneva, Switzerland: Author.

Wright, F. D., Beck, A. T., Newman, C. F., \& Liese, B. S. (1993). Cognitive therapy of substance abuse: Theoretical rationale. NIDA Research Monograph, 137, 123-146. 